\title{
IMPROVEMENTS OF THE FIVE HALVES THEOREM OF J. BOARDMAN WITH RESPECT TO THE DECOMPOSABILITY DEGREE*
}

\author{
PATRICIA E. DESIDERI ${ }^{\dagger}$ AND PEDRO L. Q. PERGHER ${ }^{\dagger}$
}

\begin{abstract}
Let $\left(M^{m}, T\right)$ be a smooth involution on a closed smooth $m$-dimensional manifold and $F=\bigcup_{j=0}^{n} F^{j}(n<m)$ its fixed point set, where $F^{j}$ denotes the union of those components of $F$ having dimension $j$. The famous Five Halves Theorem of J. Boardman, announced in 1967, establishes that, if $F$ is nonbounding, then $m \leq \frac{5}{2} n$; further, this estimative is best possible. In this paper we obtain improvements of this theorem, taking into account certain natural numbers which we call the decomposability degrees $\ell\left(F^{j}\right)$ of the nonbounding components $F^{j}$ of $F$ (see the definition in Section 1). Also, these improvements are obtained under assumptions on the set of dimensions occurring in $F$, which we denote $\pi_{0}(F)$. The main result of this paper is: suppose the involution $\left(M^{m}, T\right)$ has $\pi_{0}(F)=\{0,1, \ldots, j, n\}$, where $2 \leq j<n<m$ and $F^{j}$ is nonbounding. Write $\mathcal{M}(n-j)$ for the function of $n-j$ defined in the following way: writing $n-j=2^{p} q$, where $q \geq 1$ is odd and $p \geq 0, \mathcal{M}(n-j)=2 n+p-q+1$ if $p \leq q$ and $\mathcal{M}(n-j)=2 n+2^{p-q}$ if $p \geq q$. Then $m \leq \mathcal{M}(n-j)+2 j+\ell\left(F^{j}\right)$. In addition, we develop a method to construct involutions $\left(M^{m}, T\right)$ with $\pi_{0}(F)$ as above, in some special situations, which in some cases will show that the above bound is best possible. This will provide some improvements of the general Five Halves Theorem $\left(\pi_{0}(F)=\{i / 0 \leq i \leq n\}\right)$, by considering the particular case $j=n-1$.
\end{abstract}

Key words. Involution, Five Halves Theorem, projective space bundle, indecomposable manifold, decomposability degree, splitting principle, Stiefel-Whitney class, non-dyadic partition.

AMS subject classifications. Primary 57R85; Secondary 57R75.

1. Introduction. Suppose $M^{m}$ is a closed smooth $m$-dimensional manifold and $T: M^{m} \rightarrow M^{m}$ is a smooth involution on $M^{m}$. The fixed point set of $T, F$, can be expressed as a union of submanifolds of $M^{m}, F=\bigcup_{j=0}^{n} F^{j}$, where $F^{j}$ denotes the union of those components of $F$ having dimension $j$, and thus $n$ is the dimension of the components of $F$ of largest dimension. In our context, each $j$-dimensional part of $F$ can be assumed to be connected, since any involution is equivariantly cobordant to an involution with this property. If the involution pair $\left(M^{m}, T\right)$ is not an equivariant boundary, then $n$ cannot be too small with respect to $m$. This intriguing fact was firstly evidenced from an old result (1964) of P. E. Conner and E. E. Floyd (Theorem 27.1 of [6]), which stated: for each natural number $n$, there exists a number $\varphi(n)$ with the property that, if $\left(M^{m}, T\right)$ is an involution fixing $F=\bigcup_{j=0}^{n} F^{j}$ and if $m>\varphi(n)$, then $\left(M^{m}, T\right)$ bounds equivariantly. Later (1967), this was explicitly confirmed by the famous

Five Halves Theorem of J. Boardman: If $\left(M^{m}, T\right)$ is an involution fixing the set $F=\bigcup_{j=0}^{n} F^{j}$, for which $F$ is nonbounding (which means that at least one $F^{j}$ is

*Received September 6, 2011; accepted for publication March 1, 2013. The authors were partially supported by CNPq and FAPESP.

†Departamento de Matemática, Universidade Federal de São Carlos, Patricia E. Desideri, Caixa Postal 676, São Carlos, SP 13565-905, Brazil (patricia.desideri@gmail.com; pergher@dm.ufscar.br). 
nonbounding), then $m \leq \frac{5}{2} n .{ }^{1}$

The generality of the Five Halves Theorem, which is valid for every $n \geq 1$, allows the possibility that fixed components of all dimensions $j, 0 \leq j \leq n$, occur; in this way, it is natural to ask whether there exist better bounds for $m$ when we omit some components of $F$ and restrict the set of involved dimensions $n$. This question is inspired by the following results of the literature (as in $\operatorname{Abstract,} \pi_{0}(F)$ means the set of dimensions occurring in $F$ ):

1) (R. E. Stong and C. Kosniowski, [13], 1978): if $\left(M^{m}, T\right)$ is an involution with $\pi_{0}(F)=\{n\}$, and if $m>2 n$, then $\left(M^{m}, T\right)$ bounds equivariantly. Consequently, if $\pi_{0}(F)=\{n\}$ and $F^{n}$ is nonbounding, then $m \leq 2 n$. For each fixed $n$, with the exception of the dimensions $n=1$ and $n=3$, the maximal value $m=2 n$ is achieved by taking the involution $\left(F^{n} \times F^{n}, T\right)$, where $F^{n}$ is any nonbounding $n$-dimensional manifold and $T$ is the twist involution: $T(x, y)=(y, x)$. In other words, one has in this case an improvement for the Boardman bound by omitting the $j$-dimensional components of $F$ with $j<n$ and excluding $n=1$ and 3 .

2) (D. C. Royster, [21], Theorem 2.3, page 269, 1980): in this case, the result in question is referring to an intriguing improvement for the Boardman bound, given by $n$ odd and the omission of the $j$-dimensional components of $F$, with $0<j<n$. Let $\left(M^{m}, T\right)$ be an involution with $\pi_{0}(F)=\{0, n\}$ and $n$ odd. Then, in this case, the bound for the codimension of the top dimensional component of $F$ is constant and quite small: $m \leq n+1$. Evidently, this bound is best possible and is realized by the involution $\left(R P^{n+1}, T\right)$, where $R P^{n+1}$ is the $(n+1)$-dimensional real projective space and $T\left[x_{0}, x_{1}, \ldots, x_{n+1}\right]=\left[-x_{0}, x_{1}, \ldots, x_{n+1}\right]$, with $n$ odd.

This class of problems was introduced by P. Pergher in [16], where the case $\pi_{0}(F)=\{0, n\}$ was enlarged in the following way: if $\left(M^{m}, T\right)$ is an involution with $\pi_{0}(F)=\{0, n\}$, where $n$ is an even number having the form $n=2 p$ with $p$ odd, then $m \leq n+p+3$. This case $\left(\pi_{0}(F)=\{0, n\}\right)$ was completed by R. Stong and P. Pergher in [20]: for each natural number $n \geq 1$, if $\left(M^{m}, T\right)$ is an involution with $\pi_{0}(F)=\{0, n\}$, then $m \leq \mathcal{M}(n)$ (see Abstract for the definition of $\mathcal{M}(n)$ ). Further, this bound is best possible.

The references [7], [8], [9], [10], [11] and [12] deal with the cases $\pi_{0}(F)=\{1, n\}$, $\pi_{0}(F)=\{2, n\}$ and $\pi_{0}(F)=\{n-1, n\}$. We remark that the method used for $\pi_{0}(F)=\{n-1, n\}$ does not work for $\pi_{0}(F)=\{n-2, n\}$, and the arguments used for $\pi_{0}(F)=\{0, n\}, \pi_{0}(F)=\{1, n\}$ and $\pi_{0}(F)=\{2, n\}$ become an unpleasant mess for $\pi_{0}(F)=\{j, n\}$ if $n>j>2$. In other words, the general case $\pi_{0}(F)=\{j, n\}, n>j$, is difficult. Recently, the following advance was obtained in [17]:

Theorem A. Let $\left(M^{m}, T\right)$ be an involution with $\pi_{0}(F)=\{j, n\}$, where $F^{j}$ is indecomposable and $n>j$. Then $m \leq \mathcal{M}(n-j)+2 j+1$. Further, there are involutions with $m=\mathcal{M}(n-j)+2 j$ and $\pi_{0}(F)=\{j, n\}, n>j$, where $F^{j}$ is indecomposable, for every $n \geq 3$ and $j \geq 2$ not of the form $2^{t}-1 .^{2}$

\footnotetext{
${ }^{1}$ In fact, the first version of the Five Halves Theorem was proved under the hypothesis that $M^{m}$ is nonbounding; the result in question is a consequence of the following version obtained by $\mathrm{C}$. Kosniowski and R. E. Stong in [13]: if $\left(M^{m}, T\right)$ is a nonbounding involution, then $m \leq \frac{5}{2} n$. Further, this bound is best possible (more detailed proofs for the Five Halves Theorem can be found in [1] and $[14])$.

${ }^{2} \mathrm{~A}$ closed manifold is called indecomposable if its unoriented cobordism class cannot be expressed as a sum of products of lower dimensional cobordism classes; indecomposable $j$-dimensional manifolds occur only for $j \geq 2$ not of the form $2^{t}-1$.
} 
The decomposability hypothesis can be placed within the broader context of associating to closed manifolds its decomposability degree. To state it, write $W\left(F^{n}\right)=$ $1+w_{1}+\ldots+w_{n} \in H^{*}\left(F^{n}, Z_{2}\right)$ for the total Stiefel-Whitney class of $F^{n}$. From a given homogeneous symmetric polynomial over $Z_{2}$ of degree $n$ on degree one variables $x_{1}, x_{2}, \ldots, x_{n}, P\left(x_{1}, x_{2}, \ldots, x_{n}\right)$, we get a cohomology class in $H^{n}\left(F^{n}, Z_{2}\right)$ by identifying each $w_{i}$ to the $i$ th elementary symmetric function in the variables $x_{1}, x_{2}, \ldots, x_{n}$, and next by expressing $P\left(x_{1}, x_{2}, \ldots, x_{n}\right)$ as an $n$-dimensional polynomial in the $w_{i^{\prime} s}$. For a partition $\omega=\left(i_{1}, i_{2}, \ldots, i_{r}\right)$ of $n, 1 \leq r \leq n$, let $s_{\omega}\left(x_{1}, x_{2}, \ldots, x_{n}\right)$ be the smallest symmetric polynomial containing the monomial $x_{1}^{i_{1}} x_{2}^{i_{2}} \ldots x_{r}^{i_{r}}$ (these are the monomial symmetric functions associated to sequences $\omega$ in the literature of symmetric functions), and denote by $s_{\omega}\left(F^{n}\right) \in H^{n}\left(F^{n}, Z_{2}\right)$ the cohomology class which corresponds to $s_{\omega}\left(x_{1}, x_{2}, \ldots, x_{n}\right)$ through the previous procedure. It is well known that the cobordism class of $F^{n}$ is determined by the set of modulo 2 numbers obtained by evaluating such $n$-dimensional $Z_{2}$-cohomology classes on the fundamental homology class $\left[F^{n}\right] \in H_{n}\left(F^{n}, Z_{2}\right), s_{\omega}\left(F^{n}\right)\left[F^{n}\right]$. In fact, we do not need all of these numbers: we say that a partition $\omega=\left(i_{1}, i_{2}, \ldots, i_{r}\right)$ is non-dyadic if none of the $i_{t}$ is of the form $2^{p}-1$. From [13, Section 5], one has the following

FACT. The cobordism class of $F^{n}$ is determined by the set of numbers of the form $s_{\omega}\left(F^{n}\right)\left[F^{n}\right]$, with $\omega$ non-dyadic.

Definition. If $F^{n}$ is nonbounding, we call the decomposability degree of $F^{n}$, denoted by $\ell\left(F^{n}\right)$, the minimum length of a non-dyadic partition $\omega$ with $s_{\omega}\left(F^{n}\right)\left[F^{n}\right] \neq$ 0 (here, length $\left(\omega=\left(i_{1}, i_{2}, \ldots, i_{r}\right)\right)=l(\omega)=r$ ).

For example, if $n=2 k$ and $F^{n}$ is cobordant to $R P^{2} \times R P^{2} \times \ldots \times R P^{2}$, the cartesian product of $k$ copies of the 2-dimensional projective space $R P^{2}$, then $\ell\left(F^{n}\right)=k$. If $n \geq 2$ is not of the form $2^{t}-1$ and $F^{n}$ is indecomposable, then $\ell\left(F^{n}\right)=1$. So $\ell\left(F^{n}\right)$ is, up to cobordism, a measure of how $F^{n}$ is decomposable. From a different perspective, we can consider the question of finding bounds for the general case $\pi_{0}(F)=\{i / 0 \leq$ $i \leq n\}$, taking into account the decomposability degrees $\ell\left(F^{j}\right), 2 \leq j \leq n$. In this direction, from [18] one has the following result: if $\left(M^{m}, T\right)$ is an involution with $\pi_{0}(F)=\{i / 0 \leq i \leq n\}$ and such that the top dimensional component $F^{n}$ is indecomposable, then $m \leq 2 n+1$. Further, this bound is best possible. Generalizing this result, one has from [19] the following

Theorem B. Let $\left(M^{m}, T\right)$ be an involution with $\pi_{0}(F)=\{i / 0 \leq i \leq n\}$ and such that the top dimensional component $F^{n}$ is nonbounding. Then $m \leq 2 n+\ell\left(F^{n}\right)$. Further, this bound is best possible.

The main result of this paper generalizes Theorem A, replacing indecomposability by $\ell\left(F^{j}\right)$; further, it allows components $F^{k}$ with $0 \leq k<j$. Specifically, we prove the following

TheOREM 2.1. Let $\left(M^{m}, T\right)$ be an involution with $\pi_{0}(F)=\{0,1, \ldots, j, n\}$, where $2 \leq j<n<m$ and $F^{j}$ is nonbounding. Then $m \leq \mathcal{M}(n-j)+2 j+\ell\left(F^{j}\right)$.

In addition, given a partition $\omega=\left(i_{1}, i_{2}, \ldots, i_{t}\right)$ of $j$, we develop a method (Theorem 3.1) to construct involutions $\left(M^{m}, T\right)$ with $\pi_{0}(F)$ as above, where $m=$ $\mathcal{M}(n-j)+2 j+t$ and $s_{\omega}\left[F^{j}\right] \neq 0$, for special values of $n, j$ and $\omega$. In some special situations, this method will show that the bound given by Theorem 2.1 is best possible.

The paper is organized as follows. In Section 2 we prove the main result, Theorem 2.1 , using the Conner and Floyd theory of [6] and some special polynomials in the 
characteristic classes of total spaces of projective space bundles, introduced by Stong and Pergher in [20]. In Section 3 we develop the method mentioned above of building special involutions (Theorem 3.1); to do this, we combine some special constructions with a nontrivial result of Richard L. W. Brown of [5] and the maximal involutions of Stong and Pergher of [20].

The fact that components $F^{k}$ with $0 \leq k<j$ are allowed, together with the method above of building involutions, provides some additional improvements of the general Five Halves Theorem $\left(\pi_{0}(F)=\{i / 0 \leq i \leq n\}\right)$, by considering the particular case $j=n-1$. These improvements are direct consequences of the previous results, and will be the content of Section 4 .

2. Proof of the main result. This section will be devoted to the proof of Theorem 2.1. Take $\omega=\left(i_{1}, i_{2}, \ldots, i_{t}\right)$ a non-dyadic partition of $j$, with $s_{\omega}\left(F^{j}\right)\left[F^{j}\right] \neq 0$ and minimal length; that is, $\ell\left(F^{j}\right)=t$. For $0 \leq k \leq j$ and $k=n$, denote by $\eta_{k}$ the normal bundle of $F^{k}$ in $M^{m}$, with $\operatorname{dim}\left(\eta_{k}\right)=m-k$, and by $E_{k}$ the total space of the projective space bundle $R P\left(\eta_{k}\right)$ over $F^{k} ; E_{k}$ is a smooth closed manifold with dimension $m-1$. Denote by $\lambda_{k}$ the line bundle over $E_{k}$ associated to the double cover $S\left(\eta_{k}\right) \mapsto E_{k}$, where $S\left(\eta_{k}\right)$ is the total space of the sphere bundle associated to $\eta_{k}$. If $\eta$ is a vector bundle over a closed manifold $F$, write $W(\eta)=1+w_{1}(\eta)+w_{2}(\eta)+\ldots$ for the total Stiefel-Whitney class of $\eta$, and, as in Section $1, W(F)=1+w_{1}(F)+w_{2}(F)+\ldots$ for the Stiefel-Whitney class of the tangent bundle of $F$. From [6], one has the following algebraic tool: let $P\left(w_{1}, w_{2}, \ldots, w_{m-1} ; c\right)$ be any homogeneous polynomial over $Z_{2}$ with degree $m-1$, where each variable $w_{i}$ has degree $i$ and the variable $c$ has degree 1. For simplicity, set $w_{*}=\left(w_{1}, w_{2}, \ldots, w_{m-1}\right)$. For each $k, 0 \leq k \leq j$ or $k=n$, we can evaluate the cohomology class $P\left(w_{*}\left(E_{k}\right) ; w_{1}\left(\lambda_{k}\right)\right) \in H^{m-1}\left(E_{k}, \bar{Z}_{2}\right)$ on the fundamental homology class $\left[E_{k}\right] \in H_{m-1}\left(E_{k}, Z_{2}\right)$, thus getting the characteristic number $P\left(w_{*}\left(E_{k}\right) ; w_{1}\left(\lambda_{k}\right)\right)\left[E_{k}\right] \in Z_{2}$.

LEMMA 2.1. ([6]) The following relation is true:

$$
\sum_{k=0}^{j} P\left(w_{*}\left(E_{k}\right) ; w_{1}\left(\lambda_{k}\right)\right)\left[E_{k}\right]=P\left(w_{*}\left(E_{n}\right) ; w_{1}\left(\lambda_{k}\right)\right)\left[E_{n}\right]
$$

This follows from the fact that the disjoint union of line bundles $\left(\bigcup_{k=0}^{j} \lambda_{k}\right) \cup \lambda_{n}$ bounds as a line bundle, that is, as an element of the cobordism group of manifolds with line bundles, $\mathcal{N}_{m-1}(B O(1))$. The crucial point of our argument is the use of Lemma 2.1 with the choice of a very special polynomial $P\left(w_{*} ; c\right)$. This polynomial is the product of two special polynomials. One of them, called $\mathcal{X}$, was introduced by Stong and Pergher in [20], and has dimension $\mathcal{M}(n-j)$. The other polynomial, $\mathcal{S}_{\omega}$, associated to line bundles over closed manifolds, is built with the use of the splitting principle and is related with the partition $\omega=\left(i_{1}, i_{2}, \ldots, i_{t}\right)$. The dimension of $\mathcal{S}_{\omega}$ is $2 j+t$. Our strategy is described as follows: we suppose by contradiction that $m>\mathcal{M}(n-j)+2 j+t$, which gives $\operatorname{dim}\left(\mathcal{X} . \mathcal{S}_{\omega}\right)=\mathcal{M}(n-j)+2 j+t \leq m-1$. Then it makes sense to consider the polynomial $P=P\left(w_{*} ; c\right)=\mathcal{X} \cdot \mathcal{S}_{\omega} \cdot c^{m-1-\mathcal{M}(n-j)-2 j-t}$, with dimension $m-1$. We will have $P\left[E_{j}\right]=1$ and $P\left[E_{k}\right]=0$ for $0 \leq k<j$ and $k=n$, and thus Lemma 2.1 will give a contradiction.

We then describe the technical details of the above argument, which will be divided into some lemmas. First we describe the polynomial $\mathcal{X}$ of Stong and Pergher. Let $\eta$ be a $s$-dimensional vector bundle over a closed $b$-dimensional manifold $F$, with 
$s+b=m$, and $\lambda$ the usual line bundle over $E$, where $E$ is the total space of the projective space bundle associated to $\eta$. Set $w_{1}(\lambda)=c$. From [4], one has that

$$
W(E)=W(F) \cdot\left(\sum_{0 \leq i \leq s}(1+c)^{s-i} w_{i}(\eta)\right)
$$

For any integer $r$, one lets

$$
W[r]=1+W[r]_{1}+W[r]_{2}+\ldots=\frac{W(E)}{(1+c)^{s-r}} .
$$

Note that each class $W[r]_{u}$ is a polynomial in the classes $w_{i}(E)$ and $c$. Further, these classes satisfy the following special properties (see [20], Section 2):

$W[r]_{2 r}=w_{r}(F) c^{r}+$ terms with smaller powers of $c$,

$W[r]_{2 r+1}=\left(w_{r+1}(F)+w_{r+1}(\eta)\right) c^{r}+$ terms with smaller powers of $c$.

The polynomial $\mathcal{X}$ is a product of classes $W[r]_{u}$, considering $s=m-n$ (and so $b=n$ ) and the following list of values of $r$ : write $n-j=2^{p} q$, where $p \geq 1$ and $q$ is odd, and set $r_{i}=2^{p}-2^{p-i}$, for $1 \leq i \leq p$. Specifically, if $p<q+1$,

$$
\mathcal{X}=W\left[r_{p}\right]_{2^{p+1}-1}^{q+1-p} \cdot \prod_{1 \leq i \leq p} W\left[r_{i}\right]_{2 r_{i}}
$$

and if $p \geq q+1$

$$
\mathcal{X}=\prod_{1 \leq i \leq q+1} W\left[r_{i}\right]_{2 r_{i}}
$$

An easy calculation shows that $\mathcal{X}$ has dimension $\mathcal{M}(n-j)$; also, by using the properties of the classes $W[r]_{u}$ above listed, it can be proved that $\mathcal{X}$ has the form

$$
\mathcal{X}=A_{y} c^{\mathcal{M}(n-j)-y}+\text { terms with smaller powers of } c,
$$

where $A_{y}$ is a cohomology class of dimension $y \geq n-j+1$ and comes from the cohomology of $F$ (see [9] or [20]).

Now we describe the polynomial $\mathcal{S}_{\omega}$, which is associated to a line bundle $\lambda$ over a closed $(m-1)$-dimensional manifold $N$. Let $\mathcal{S}_{\omega}\left(x_{1}, x_{2}, \ldots, x_{m-1}, c\right)$ be the smallest polynomial on degree one variables $x_{1}, x_{2}, \ldots, x_{m-1}, c$, which is symmetric in the variables $x_{1}, x_{2}, \ldots, x_{m-1}$ and contains the polynomial

$$
x_{1}^{i_{1}}\left(x_{1}+c\right)^{i_{1}+1} x_{2}^{i_{2}}\left(x_{2}+c\right)^{i_{2}+1} \ldots x_{t}^{i_{t}}\left(x_{t}+c\right)^{i_{t}+1} .
$$

We then identify $w_{1}(\lambda)$ to $c$ and each $w_{i}(N)$ to the $i$ th elementary symmetric function in the variables $x_{1}, x_{2}, \ldots, x_{m-1}$; next, we express $\mathcal{S}_{\omega}\left(x_{1}, x_{2}, \ldots, x_{m-1}, c\right)$ as a polynomial of dimension $2 j+t$ in the $w_{i}^{\prime} s(N)$ and $w_{1}(\lambda)$. It is convenient to denote this polynomial by $\mathcal{S}_{\omega}(\lambda)$. The crucial point is that $\mathcal{S}_{\omega}(\lambda)$ has a nice behavior with respect to the standard line bundles over the total spaces of projective space bundles.

Lemma 2.2. As before, let $\eta$ be a s-dimensional vector bundle over a closed $b$ dimensional manifold $F$, with $s+b=m, \lambda$ the usual line bundle over $E$, where $E$ is the total space of the projective space bundle associated to $\eta$, and $w_{1}(\lambda)=c$. Then

$$
\mathcal{S}_{\omega}(\lambda)=s_{\omega}(F) c^{j+t}+\text { terms with smaller powers of } c .
$$


Proof. We use the splitting principle, which allows to write the Stiefel-Whitney class of any $s$-dimensional vector bundle formally as $1+w_{1}+w_{2}+\ldots+w_{s}=(1+$ $\left.x_{1}\right)\left(1+x_{2}\right) \ldots\left(1+x_{s}\right)$, where each $x_{i}$ has degree one, and effectively to see each $w_{i}$ as the $i$ th elementary symmetric function in the variables $x_{1}, x_{2}, \ldots, x_{s}$. Write $W(F)=\left(1+x_{1}\right)\left(1+x_{2}\right) \ldots\left(1+x_{b}\right)$ and $W(\eta)=\left(1+y_{1}\right)\left(1+y_{2}\right) \ldots\left(1+y_{s}\right)$. Then

$$
W(E)=\left(1+x_{1}\right)\left(1+x_{2}\right) \ldots\left(1+x_{b}\right)\left(1+c+y_{1}\right)\left(1+c+y_{2}\right) \ldots\left(1+c+y_{s}\right) .
$$

First we collect the terms with greatest power of $c$, and to do this we must analyze terms

$$
z_{e_{1}}^{i_{1}}\left(z_{e_{1}}+c\right)^{i_{1}+1} z_{e_{2}}^{i_{2}}\left(z_{e_{2}}+c\right)^{i_{2}+1} \ldots z_{e_{t}}^{i_{t}}\left(z_{e_{t}}+c\right)^{i_{t}+1}
$$

coming from ordered subsets of degree one variables

$$
\begin{aligned}
& \left\{z_{e_{1}}, z_{e_{2}}, \ldots, z_{e_{t}}\right\} \subset\left\{z_{1}, z_{2}, \ldots, z_{m}\right\}=\left\{x_{1}, x_{2}, \ldots, x_{b}, y_{1}+c, y_{2}+c, \ldots, y_{s}+c\right\} . \\
& \text { If }\left\{z_{e_{1}}, z_{e_{2}}, \ldots, z_{e_{t}}\right\} \subset\left\{x_{1}, x_{2}, \ldots, x_{b}\right\}, \text { we can write } \\
& z_{e_{1}}^{i_{1}}\left(z_{e_{1}}+c\right)^{i_{1}+1} z_{e_{2}}^{i_{2}}\left(z_{e_{2}}+c\right)^{i_{2}+1} \ldots z_{e_{t}}^{i_{t}}\left(z_{e_{t}}+c\right)^{i_{t}+1}= \\
& =z_{e_{1}}^{i_{1}}\left(c^{i_{1}+1}+\sum_{f=0}^{i_{1}}\left(\begin{array}{c}
i_{1}+1 \\
f
\end{array}\right) z_{e_{1}}^{i_{1}+1-f} c^{f}\right) \ldots z_{e_{t}}^{i_{t}}\left(c^{i_{t}+1}+\sum_{f=0}^{i_{t}}\left(\begin{array}{c}
i_{t}+1 \\
f
\end{array}\right) z_{e_{t}}^{i_{t}+1-f} c^{f}\right)= \\
& =z_{e_{1}}^{i_{1}} z_{e_{2}}^{i_{2}} \ldots z_{e_{t}}^{i_{t}} c^{j+t}+\text { terms with smaller powers of } c .
\end{aligned}
$$

Therefore, the sum of all terms coming from subsets

$$
\left\{z_{e_{1}}, z_{e_{2}}, \ldots, z_{e_{t}}\right\} \subset\left\{x_{1}, x_{2}, \ldots, x_{b}\right\}
$$

has the form $s_{\omega}(F) c^{j+t}+$ terms with smaller powers of $c$, with the agreement that the cohomology class $s_{\omega}(F) \in H^{j}\left(F, Z_{2}\right)$ makes sense also for $j \neq b$.

If $\left\{z_{e_{1}}, z_{e_{2}}, \ldots, z_{e_{t}}\right\}$ has $x$ elements of the set $\left\{y_{1}+c, y_{2}+c, \ldots, y_{s}+c\right\}, 1 \leq x \leq t$, the same calculation above shows that $c^{j+t-x}$ is the greatest power of $c$ that appears in the terms coming from $\left\{z_{e_{1}}, z_{e_{2}}, \ldots, z_{e_{t}}\right\}$. Putting together the two possibilities, the lemma then follows.

Following our strategy, the next step is to calculate $P\left[E_{k}\right]$ for $0 \leq k \leq j$ and $k=n$, where $P=\mathcal{X} \cdot \mathcal{S}_{\omega}\left(\lambda_{k}\right) \cdot c^{m-1-\mathcal{M}(n-j)-2 j-t}$.

Lemma 2.3. One has $P\left[E_{n}\right]=0$.

Proof. As before mentioned, $\mathcal{X}=A_{y} c^{\mathcal{M}(n-j)-y}+$ terms with smaller powers of $c$, where $A_{y}$ is a cohomology class of dimension $y \geq n-j+1$ and comes from the cohomology of $F^{n}$. Thus each term of $\mathcal{X}$ has a factor of dimension at least $n-j+1$ from the cohomology of $F^{n}$. On the other hand, Lemma 2.2 says that every term of $\mathcal{S}_{\omega}\left(\lambda_{n}\right)$ has a factor of dimension at least $j$ from the cohomology of $F^{n}$. In this way, $\mathcal{X} . \mathcal{S}_{\omega}\left(\lambda_{n}\right)$ is a class in $H^{\mathcal{M}(n-j)+2 j+t}\left(E_{n}, Z_{2}\right)$ with each one of its terms having a factor of dimension at least $n+1$ from $F^{n}$, which means that $\mathcal{X} \cdot \mathcal{S}_{\omega}\left(\lambda_{n}\right)=0$. Then $P\left[E_{n}\right]=0$, as desired.

Lemma 2.4. For $0 \leq k<j$, one has that $P\left[E_{k}\right]=0$. 
Proof. This follows from Lemma 2.2 and the fact that $s_{\omega}\left(F^{k}\right)$ is a cohomology class of dimension $j>k$ coming from the cohomology of $F^{k}$.

LEMMA 2.5. One has $P\left[E_{j}\right]=1$.

Proof. From Lemma 2.2, one has

$$
\begin{aligned}
\mathcal{S}_{\omega}\left(\lambda_{j}\right) & =s_{\omega}\left(F^{j}\right) c^{j+t}+\text { terms with smaller powers of } c= \\
& =s_{\omega}\left(F^{j}\right) c^{j+t}+\sum A_{y} c^{x}
\end{aligned}
$$

where each $A_{y}$ is a cohomology class of dimension $y$ coming from $F^{j}$, with $y+x=2 j+t$ and $x<j+t$. Thus, $y>j$ and so $A_{y}=0$, which gives $\mathcal{S}_{\omega}\left(\lambda_{j}\right)=s_{\omega}\left(F^{j}\right) c^{j+t}$. This implies that, if $I$ denotes the ideal of $H^{*}\left(E_{j}, Z_{2}\right)$ generated by the classes coming from $F^{j}$ and with positive dimension, then $\mathcal{S}_{\omega}\left(\lambda_{j}\right) \cdot \theta=0$, for each $\theta \in I$. Thus, in the computation of $\mathcal{X}$ for $k=j$, one needs to consider only that $W\left(E_{j}\right) \equiv(1+c)^{m-j}$ $\bmod I$. Further, $\mathcal{X}$ is obtained with $s=m-n$, which means that in this case each factor $W[r]$ of $\mathcal{X}$ takes the form

$$
W[r]=\frac{W\left(E_{j}\right)}{(1+c)^{m-n-r}} \equiv(1+c)^{n-j+r} \quad \bmod I=(1+c)^{2^{p} q+r} \quad \bmod I .
$$

Specializing, for $r_{i}=2^{p}-2^{p-i}, i=1,2, \ldots, p$, one has

$$
W\left[r_{i}\right]_{2 r_{i}} \equiv\left(\begin{array}{c}
2^{p} q+2^{p}-2^{p-i} \\
2^{p+1}-2^{p-i+1}
\end{array}\right) c^{2 r_{i}} \bmod I,
$$

and for $r=2^{p}-1$, one has

$$
W[r]_{2 r+1} \equiv\left(\begin{array}{c}
2^{p} q+2^{p}-1 \\
2^{p+1}-1
\end{array}\right) c^{2 r+1} \bmod I .
$$

The lesser term of the 2-adic expansion of $2^{p} q+2^{p}$ is $2^{p+1}$. Using the fact that a binomial coefficient $\left(\begin{array}{l}a \\ b\end{array}\right)$ is nonzero modulo 2 if and only if the 2 -adic expansion of $b$ is a subset of the 2-adic expansion of $a$, we conclude that the above binomial coefficients are nonzero modulo 2. It follows that each factor $W[r]_{u}$ of $\mathcal{X}$ satisfies $W[r]_{u} \equiv c^{u}$ $\bmod I$, which implies that $\mathcal{X} \equiv c^{\mathcal{M}(n-j)} \bmod I$. Thus

$$
P=\mathcal{X} \cdot \mathcal{S}_{\omega}\left(\lambda_{j}\right) \cdot c^{m-1-\mathcal{M}(n-j)-2 j-t}=s_{\omega}\left(F^{j}\right) \cdot c^{m-j-1} .
$$

Since from the Leray-Hirsch Theorem (see [3]; p. 129), $H^{*}\left(E_{j}, Z_{2}\right)$ is the free $H^{*}\left(F^{j}, Z_{2}\right)$-module on $1, c, c^{2}, \ldots, c^{m-j-1}$, we conclude that

$$
P\left[E_{j}\right]=s_{\omega}\left(F^{j}\right) \cdot c^{m-j-1}\left[E_{j}\right]=s_{\omega}\left(F^{j}\right)\left[F^{j}\right]=1 .
$$

As previously explained, Theorem 2.1 then is established.

3. Maximal involutions. Take natural numbers $j$, $n$, with $2 \leq j<n$, and let $\omega=\left(i_{1}, i_{2}, \ldots, i_{t}\right)$ be a non-dyadic partition of $j$. As announced in Section 1 , in this section we develop a method to construct an involution $\left(M^{m}, T\right)$ whose fixed point set has the form $F=\left(\bigcup_{k<j} F^{k}\right) \cup F^{j} \cup F^{n}$, with $m=\mathcal{M}(n-j)+2 j+t$ and $s_{\omega}\left[F^{j}\right] \neq 0$, for special values of $n, j$ and $\omega$ (for technical reasons, in this section we abandon the notation $\pi_{0}(F)$ ). To perform this construction, we need the following facts: 
Fact A (R. L. W. Brown, [5]). Let $P^{j}$ be a closed smooth $j$-dimensional manifold, and consider the orbit space $\frac{S^{1} \times P^{j} \times P^{j}}{-1 \times \text { twist }}$, which is a closed $2 j+1$-dimensional manifold; here, $S^{1}$ is the unit circle. Then, if $P^{j}$ is indecomposable, $\frac{S^{1} \times P^{j} \times P^{j}}{-1 \times t w i s t}$ also is.

Fact B (R. Stong and P. Pergher, [20]). There are involutions $\left(N^{m}, S\right)$ having fixed point set of the form $\{$ point $\} \cup F^{n}$, with $m=\mathcal{M}(n)$, for every $n \geq 1$.

Fact C (P. E. Conner and E. E. Floyd, $[6])$. Let $\left(W^{n}, T\right)$ be an involution defined on a closed smooth $n$-dimensional manifold $W^{n}$, with fixed point set $F$. Suppose that $W^{n}$ bounds. Then the involution $\Gamma\left(W^{n}, T\right)=\left(\frac{S^{1} \times W^{n}}{-1 \times T}, \tau\right)$, defined on the orbit space $\frac{S^{1} \times W^{n}}{-1 \times T}$ (which has dimension $n+1$ ), is equivariantly cobordant to an involution $\left(N^{n+1}, S\right)$ fixing $F$; here, $\tau$ is the involution induced by $c \times 1$, where $c$ is the complex conjugation. If the orbit space $\frac{S^{1} \times W^{n}}{-1 \times T}$ is a boundary, we can repeat the process taking $\Gamma^{2}\left(W^{n}, T\right)$, and so on.

Fact D (P. Pergher, A. Ramos and R. Oliveira, [15]). For $m, n$ even, $m<n$, write $n-m=2^{p} q$, where $p \geq 1$ and $q \geq 1$ is odd. Let $\left(R P^{m+n+1}, T_{m, n}\right)$ be the involution

$$
T_{m, n}\left[x_{0}, x_{1}, \ldots, x_{m+n+1}\right]=\left[-x_{0},-x_{1}, \ldots,-x_{m}, x_{m+1}, \ldots, x_{m+n+1}\right],
$$

whose fixed point set is $F=R P^{m} \cup R P^{n}$. Then the underlying manifold of the involution $\Gamma^{j}\left(R P^{m+n+1}, T_{m, n}\right)$ bounds for $0 \leq j \leq 1$ if $p=1$, and for $0 \leq j \leq 2^{p}-2$ if $p>1$. From Fact $\mathrm{C}$, this gives that $\Gamma^{j}\left(R P^{m+n+1}, T_{m, n}\right)$ is cobordant to an involution $\left(M^{m+n+1+j}, T\right)$ fixing $F=R P^{m} \cup R P^{n}$, for $0<j \leq 2$ if $p=1$, and for $0<j \leq 2^{p}-1$ if $p>1$.

The following two lemmas are crucial for the desired construction.

Lemma 3.1. Suppose that $\left(M^{m}, T\right)$ is an involution with fixed point set of the form $P^{j} \cup F^{n}$, where $j \neq n$ and $P^{j}$ is indecomposable. There is then an involution $\left(W^{2 m+1}, T^{\prime}\right)$ whose fixed point set has the form $P^{2 j+1} \cup F^{2 n+1}$, where $P^{2 j+1}$ is indecomposable.

Proof. On $S^{1} \times M^{m} \times M^{m}$, consider the involution $1 \times T \times T$. This involution commutes with $-1 \times$ twist and then induces an involution $S$ on the orbit space $N=\frac{S^{1} \times M^{m} \times M^{m}}{-1 \times \text { twist }}$. The fixed set of $S$ is the union of three manifolds:

$$
\underbrace{\frac{S^{1} \times P^{j} \times P^{j}}{-1 \times t w i s t}}_{P^{2 j+1}} \cup \underbrace{\frac{S^{1} \times F^{n} \times F^{n}}{-1 \times t w i s t}}_{F^{2 n+1}} \cup \frac{S^{1} \times\left(P^{j} \times F^{n} \cup F^{n} \times P^{j}\right)}{-1 \times \text { twist }} \text {. }
$$

Because $j \neq n$, there is a tubular neighborhood of $\frac{S^{1} \times\left(P^{j} \times F^{n} \cup F^{n} \times P^{j}\right)}{-1 \times \text { twist }}$ in $N$ which is diffeomorphic to a tubular neighborhood of $S^{1} \times P^{j} \times F^{n}$ in $S^{1} \times M^{m} \times M^{m}$. Then, this fixed manifold is $S^{1} \times P^{j} \times F^{n}$ with normal bundle $S^{1} \times \nu$, where $\nu$ is the normal bundle of $P^{j} \times F^{n}$ in $M^{m} \times M^{m}$ (that is, $S^{1} \times \nu$ is the pullback of $\nu$ by the projection $S^{1} \times P^{j} \times F^{n} \rightarrow P^{j} \times F^{n}$ ), and being a product with $S^{1}$ this bundle bounds. Thus, $(N, S)$ is cobordant to an involution $\left(W^{2 m+1}, T^{\prime}\right)$ with fixed point set of the form $P^{2 j+1} \cup F^{2 n+1}$, and by Fact A $P^{2 j+1}$ is indecomposable. 
LEMmA 3.2. If $j \geq 2$ is not of the form $2^{s}-1$, write $j+1=2^{p}(2 q+1)$, where $p \geq 0$ and $q>0$ (note then that $j-2^{p+1}=2^{p+1}(q-1)+2^{p}-1 \geq 0$ ). There is then an involution $\left(M^{2 j+1}, T\right)$ with fixed point set of the form $P^{j} \cup F^{n}$, where $n=j-2^{p+1}$ and $P^{j}$ is indecomposable.

Proof. We use induction on $p \geq 0$. For $p=0$, we have $j=2 q$ and $j-2^{p+1}=2 q-2$. From Fact D, the involution $\Gamma^{2}\left(R P^{4 q-1}, T_{2 q-2,2 q}\right)$ is equivariantly cobordant to an involution $\left(M^{4 q+1}, T\right)$ fixing $R P^{2 q-2} \cup R P^{2 q}$. Since $R P^{2 q}$ is indecomposable, the result is true for $p=0$.

Now suppose $\left(M^{2 j+1}, T\right)$ an involution with fixed point set of the form $P^{j} \cup F^{n}$, where $j+1=2^{p}(2 q+1)$ with $p \geq 0$ and $q>0, n=j-2^{p+1}$ and $P^{j}$ is indecomposable. From Lemma 3.1, there is an involution $\left(M^{\prime}, T^{\prime}\right)$ with $\operatorname{dim} M^{\prime}=2(2 j+1)+1$ and fixed point set of the form $P^{2 j+1} \cup F^{2 n+1}$, where $P^{2 j+1}$ is indecomposable. Since $(2 j+1)+1=2^{p+1}(2 q+1)$ and $2 n+1=(2 j+1)-2^{p+2}$, the result is proved.

We are now able to state and prove the main result of this section.

TheOREM 3.1. Let $\omega=\left(i_{1}, i_{2}, \ldots, i_{t}\right)$ be a non-dyadic partition of $j$, and for each $1 \leq r \leq t$ write $i_{r}+1=2^{p_{r}}\left(2 q_{r}+1\right)$, with $p_{r} \geq 0$ and $q_{r}>0$. Suppose that $n>j$ and $n-j<2^{p_{r}+1}$, for each $1 \leq r \leq t$. Then there is an involution $\left(M^{m}, T\right)$ with $m=\mathcal{M}(n-j)+2 j+t$ and fixed point set of the form

$$
F=\left(\bigcup_{k<j} F^{k}\right) \cup F^{j} \cup F^{n},
$$

where $s_{\omega}\left[F^{j}\right] \neq 0$.

Proof. Lemma 3.2 provides, for each $r \in\{1,2, \ldots, t\}$, an involution $\left(M^{2 i_{r}+1}\right.$, $\left.T_{r}\right)$ with fixed point set of the form $P^{i_{r}} \cup F^{i_{r}-2^{p_{r}+1}}$, where $P^{i_{r}}$ is indecomposable. Set $F^{j}=P^{i_{1}} \times P^{i_{2}} \times \ldots \times P^{i_{t}}$, and consider the set of natural numbers $\mathcal{A}=\left\{\sum_{r \in \sigma} 2^{p_{r}+1} / \sigma \subset\{1,2, \ldots, t\}, \sigma\right.$ nonempty $\}$. Consider the product involution $\left(W^{2 j+t}, U\right)=\left(M^{2 i_{1}+1}, T_{1}\right) \times\left(M^{2 i_{2}+1}, T_{2}\right) \times \ldots \times\left(M^{2 i_{t}+1}, T_{t}\right)$. The fixed point set of $\left(W^{2 j+t}, U\right)$ has the form $F^{j} \cup D$, where each component of $D$ has dimension $j-a$ for some $a \in \mathcal{A}$; conversely, for each $a \in \mathcal{A}$, there is a component of $D$ with dimension $j-a$. From Fact B, one has an involution $\left(N^{\mathcal{M}(n-j)}, S\right)$ with fixed point set of the form $\{$ point $\} \cup G^{n-j}$; set $F^{n}=F^{j} \times G^{n-j}$. Then the desired involution is $\left(M^{m}, T\right)=\left(W^{2 j+t}, U\right) \times\left(N^{\mathcal{M}(n-j)}, S\right)$. In fact, $m=\mathcal{M}(n-j)+2 j+t$ and the fixed point set of $\left(M^{m}, T\right)$ has the form $D \cup\left(D \times G^{n-j}\right) \cup F^{j} \cup F^{n}$, with the components of $D$ having dimension less than $j$; further, using the fact that each $P^{i_{r}}$ is indecomposable, the splitting principle and dimensional considerations, one has

$$
s_{\omega}\left[F^{j}\right]=s_{i_{1}}\left[P^{i_{1}}\right] s_{i_{2}}\left[P^{i_{2}}\right] \ldots s_{i_{t}}\left[P^{i_{t}}\right] \neq 0 .
$$

Thus it remains to show that the components of $D \times G^{n-j}$ have dimension less than $j$. These dimensions are of the form $(n-j)+(j-a)=n-a$, where $a \in \mathcal{A}$. If $a \in \mathcal{A}$, there is $r \in\{1,2, \ldots, t\}$ such that $a \geq 2^{p_{r}+1}$, and so $n-a \leq n-2^{p_{r}+1}$. Since by hypothesis $n-j<2^{p_{r}+1}$, one then has $n-a<j$, which ends the proof.

REMARK. For $j<n$ fixed, the bound $m \leq \mathcal{M}(n-j)+2 j+\ell\left(F^{j}\right)$ of Theorem 2.1 is then best possible for nonbounding manifolds $F^{j}$ whose decomposability degree $\ell\left(F^{j}\right)$ is realized by partitions $\omega$ satisfying the condition required by Theorem 3.1. For example, it is best possible for $j=n-1$ (see Section 4). For $j=n-2$ and $j=n-3$, it is best possible for manifolds $F^{j}$ for which $\ell\left(F^{j}\right)$ is realized by partitions $\omega=\left(i_{1}, i_{2}, \ldots, i_{t}\right)$ where $i_{r}$ is odd, $1 \leq r \leq t$. In particular, it is best possible if $j=n-2$ or $j=n-3, F^{j}$ is indecomposable and $j$ is odd. 
4. Some improvements of the Five Halves Theorem. In this section we obtain some improvements of the general Five Halves Theorem $\left(\pi_{0}(F)=\{i / 0 \leq\right.$ $i \leq n\}$ ), which are direct consequences of our previous results. First we obtain the following improvement for Theorem B (see Section 1):

Theorem 4.1. let $\left(M^{m}, T\right)$ be an involution with $\pi_{0}(F)=\{i / 0 \leq i \leq n\}$ and such that the components $F^{n}$ and $F^{n-1}$ are nonbounding. Then $m \leq \min \{2 n+$ $\left.l\left(F^{n-1}\right), 2 n+l\left(F^{n}\right)\right\}$. Further, the bounds $m \leq 2 n+\ell\left(F^{n-1}\right)$ and $m \leq 2 n+\ell\left(F^{n}\right)$ are separately best possible.

Proof. From [19], one knows that $m \leq 2 n+\ell\left(F^{n}\right)$ and, under the condition " $F$ " is nonbounding", this bound is best possible. From Theorem 2.1 with $j=n-1$ and the fact that $\mathcal{M}(1)=2$, one has

$$
m \leq \mathcal{M}(1)+2(n-1)+\ell\left(F^{n-1}\right)=2 n+\ell\left(F^{n-1}\right) .
$$

Now, if $\omega=\left(i_{1}, i_{2}, \ldots, i_{t}\right)$ is a non-dyadic partition of $n-1$, and if we write $i_{j}+1=2^{p} q$, with $p \geq 0$ and $q$ odd, certainly $2^{p+1} \geq 2$ and so $n-(n-1)=1<2^{p+1}$. This means that Theorem 3.1 gives an involution $\left(M^{m}, T\right)$ with $m=2 n+t$ and fixed point set $F=\bigcup_{j=0}^{n} F^{j}$ satisfying $s_{\omega}\left(F^{n-1}\right)\left[F^{n-1}\right] \neq 0$; in particular, this is true for a partition $\omega$ that realizes $\ell\left(F^{n-1}\right)$. This shows that, under the condition " $F^{n-1}$ is nonbounding", the bound $m \leq 2 n+\ell\left(F^{n-1}\right)$ is best possible, and Theorem 4.1 is proved.

We remark that the precise statement of the Five Halves Theorem is: if $\left(M^{m}, T\right)$ has $\pi_{0}(F)=\{i / 0 \leq i \leq n\}$ with $F$ nonbounding, then

(i) if $n=2 k$ with $k \geq 1, m \leq 5 k$, and

(ii) if $n=2 k+1$ with $k \geq 0, m \leq 5 k+2$.

We have seen in [19] that Theorem $B$ gives the following improvement in case (ii): if $\left(M^{m}, T\right)$ has $\pi_{0}(F)=\{i / 0 \leq i \leq n\}$ with $F^{n}$ nonbounding and $n=2 k+1$, then $m \leq 5 k+1$; further, this bound is best possible. In the same direction, Theorem 4.1 gives the following improvement in case (i):

TheOREM 4.2. Let $\left(M^{m}, T\right)$ be an involution with $\pi_{0}(F)=\{i / 0 \leq i \leq n\}$, where $n=2 k, k \geq 3$ and $F^{n-1}$ is nonbounding. Then $m \leq 5 k-2$, and this bound is best possible.

Proof. Since $F^{n-1}$ is nonbounding, $m \leq 2 n+\ell\left(F^{n-1}\right)$. Among all non-dyadic partitions of $n-1=2 k-1$, the maximal lenght occurs for the partition $\omega=(2,2, \ldots, 2,5)$, whose lenght is $k-2$. Then $1 \leq l\left(F^{n-1}\right) \leq k-2$ and

$$
m \leq 2 n+\ell\left(F^{n-1}\right) \leq 4 k+k-2=5 k-2 .
$$

Further, the argument of Theorem 4.1 particularized for the partition $\omega=$ $(2,2, \ldots, 2,5)$ shows that there is an involution $\left(M^{m}, T\right)$ with $m=5 k-2$ and fixed set $F=\bigcup_{j=0}^{n} F^{j}$ satisfying $s_{\omega}\left(F^{n-1}\right)\left[F^{n-1}\right] \neq 0$. Therefore $m \leq 5 k-2$ is best possible.

If $\left(M^{m}, T\right)$ has $\pi_{0}(F)=\{i / 0 \leq i \leq n\}$ with $F$ nonbounding and $t$ is the codimension of the top dimensional component $F^{n}$, then the Five Halves Theorem says that $t \leq 3 k$ if $n=2 k$, and $t \leq 3 k+1$ if $n=2 k+1$. In this way, these bounds for $t$ increase with $n$, that is, they are not limited as functions of $n$. Now, if $\left(M^{m}, T\right)$ 
has $\pi_{0}(F)=\{0,1, \ldots, j, n\}$, where $2 \leq j<n<m$ and $F^{j}$ is nonbounding, then Theorem 2.1 says that the codimension of the top dimensional component satisfies $t \leq \mathcal{M}(n-j)+2 j+\ell\left(F^{j}\right)-n$. For $j$ fixed, an easy calculation shows that, if $n-j$ is even, then $\mathcal{M}(n-j)-n$ is not limited as a function of $n$, and so in the same way the bound for $t$ increases with $n$ in this case. However, if $n-j$ is odd, one has unlike the following small codimension phenomenon:

Theorem 4.3. Suppose that $\left(M^{m}, T\right)$ has $\pi_{0}(F)=\{0,1, \ldots, j, n\}$, where $2 \leq$ $j<n<m$ and $F^{j}$ is nonbounding, and denote by $t$ the codimension of the top dimensional component $F^{n}$. Then, for $j$ fixed, $t$ is limited as a function of $n$ if $n-j$ is odd. Specifically, if $j=2 k+1$ is odd, $t \leq j+k$, and if $j=2 k$ is even, $t \leq j+k+1$.

Proof. Among all non-dyadic partitions of $j=2 k+1$, the maximal lenght occurs for the partition $\omega=(2,2, \ldots, 2,5)$, with lenght $k-1$, and for $j=2 k$ the maximal lenght is $k$. Thus, if $j=2 k+1,1 \leq l\left(F^{j}\right) \leq k-1$, and if $j=2 k, 1 \leq l\left(F^{j}\right) \leq k$. Further, if $n-j$ is odd, $\mathcal{M}(n-j)=n-j+1$. Then, if $j=2 k+1$,

$$
t \leq \mathcal{M}(n-j)+2 j+\ell\left(F^{j}\right)-n \leq n-j+1+2 j+k-1-n=j+k,
$$

and, similarly, if $j=2 k, t \leq j+k+1$.

Acknowledgements. We would like to express our gratitude to the referees for a careful reading and valuable suggestions concerning the presentation of this paper, which led to this present version.

\section{REFERENCES}

[1] J. M. Boardman, Cobordism of involutions revisited, Proceedings of the Second Conference on Compact Transformation Groups, Univ. Massachusetts, Amherst, Mass., Part I, (1971), pp. 131-151. Lecture Notes in Math., Vol. 298, Springer, Berlin (1972).

[2] J. M. Boardman, On manifolds with involution, Bulletin Amer. Math. Soc., 73 (1967), pp. 136138.

[3] A. BoRel, Sur la cohomologie des espaces fibr?s principaux et des espaces homog?nes des groupes de Lie compacts, Ann. of Math., 57:2 (1953), pp. 115-207.

[4] A. Borel And F. Hirzebruch, Characteristic classes and homogeneous spaces, I, Amer. J. Math., 80 (1958), pp. 458-538.

[5] R. L. W. Brown, Immersions and embeddings up to cobordism, Canadian J. Math., 23:6 (1971), pp. 1102-1115.

[6] P. E. Conner And E. E. Floyd, Differentiable periodic maps, Springer-Verlag, Berlin, (1964).

[7] F. G. Figueira And P. L. Q. Pergher, Bounds on the dimension of manifolds with involution fixing $F^{n} \cup F^{2}$, Glasgow Math. J., 50 (2008), pp. 595-604.

[8] F. G. Figueira and P. L. Q. Pergher, Dimensions of fixed point sets of involutions, Arch. Math. (Basel), 87:3 (2006), pp. 280-288.

[9] F. G. Figueira and P. L. Q. Pergher, Involutions fixing $F^{n} \cup F^{2}$, Topology Appl., 153:14 (2006), pp. 2499-2507.

[10] F. G. Figueira And P. L. Q. Pergher, Two commuting involutions fixing $F^{n} \cup F^{n-1}$, Geom. Dedicata, 117 (2006), pp. 181-193.

[11] S. M. Kelton, Involutions fixing $R P^{j} \cup F^{n}$, Topology Appl., 142 (2004), pp. 197-203.

[12] S. M. Kelton, Involutions fixing $R P^{j} \cup F^{n}$, II., Topology Appl., 149:1-3 (2005), pp. 217-226.

[13] C. Kosniowski And R. E. Stong, Involutions and characteristic numbers, Topology, 17 (1978), pp. 309-330.

[14] J. Morava, Cobordism of involutions revisited, revisited. Homotopy invariant algebraic structures, Baltimore, MD, (1998), pp. 15-18. Contemp. Math., 239, Amer. Math. Soc., Providence, RI, (1999).

[15] R. Oliveira, P. L. Q. Pergher, And A. Ramos, $Z_{2}^{k}$-actions fixing $R P^{2} \cup R P^{e v e n}$, Algebr. Geom. Topol., 7 (2007), pp. 29-45.

[16] P. L. Q. Pergher, Bounds on the dimension of manifolds with certain $Z_{2}$ fixed sets, Mat. Contemp., 13 (1996), pp. 269-275. 
[17] P. L. Q. Pergher, Involutions fixing $F^{n} \cup\{$ Indecomposable $\}$, Canadian Math. Bull., (2011), (DOI) 10.4153/CMB-2011-051-4.

[18] P. L. Q. Pergher, Involutions whose top dimensional component of the fixed point set is indecomposable, Geom. Dedicata, 146 (2010), pp. 1-7.

[19] P. L. Q. Pergher, An Improvement of the five halves theorem of J. Boardman, Israel Journal of Math, (2011), (DOI) 10.1007/s11856-011-0125-2.

[20] P. L. Q. Pergher And R. E. Stong, Involutions fixing $\{$ point $\} \cup F^{n}$, Transformation Groups, 6 (2001), pp. 78-85.

[21] D. C. Royster, Involutions fixing the disjoint union of two projective spaces, Indiana Univ. Math. J., 29:2 (1980), pp. 267-276.

[22] R. Thом, Quelques propriété?s globales des variétés différentiables, Comment. Math. Helv., 28:1-3 (1954), pp. 17-86. 